\title{
State-dependent Topological Invariants and Anomalous Bulk-Boundary Correspondence in non-Hermitian Topological Systems
}

\author{
Xiao-Ran Wang, ${ }^{1}$ Cui-Xian Guo, ${ }^{1}$ and Su-Peng Kou, ${ }^{1}$, \\ ${ }^{1}$ Center for Advanced Quantum Studies, Department of Physics, \\ Beijing Normal University, Beijing 100875, China
}

\begin{abstract}
The breakdown of the bulk-boundary correspondence in non-Hermitian (NH) topological systems is an open, controversial issue. In this paper, to resolve this issue, we ask the following question: Can a (global) topological invariant completely describe the topological properties of a NH system as its Hermitian counterpart? Our answer is no. One cannot use a global topological invariant (including non-Bloch topological invariant) to accurately characterize the topological properties of the NH systems. Instead, there exist a new type of topological invariants that are absence in its Hermitian counterpart - the state-dependent topological invariants. With the help of the statedependent topological invariants, we develop a new topological theory for NH topological system beyond the general knowledge for usual Hermitian systems and obtain an exact formulation of the bulk-boundary correspondence, including state-dependent phase diagram, state-dependent phase transition and anomalous transport properties (spontaneous topological current). Therefore, these results will help people to understand the exotic topological properties of various non-Hermitian systems.
\end{abstract}

Topological systems, including topological insulators and topological superconductors have become the forefront of research in condensed matter physics for many years [1 9]. These gapped topological system are always characterized by certain (global) topological invariants and have intrinsic topological properties that are robust and immunes to perturbations. For two quantum phases with different topological invariants, one cannot deform the ground states from one quantum phase to the other without closing the energy gap. On the other hand, nonHermitian (NH) topological systems have been intensively studied in both theory 10 59] and experiments 60 70. The topological properties of $\mathrm{NH}$ systems show quite different properties as their Hermitian counterparts. Recently, within the generalization of Altland-Zirnbauer (AZ) theory, the classification of NH systems with topological bands is characterized by different symmetryprotected topological invariants 21, 34, 35].

An open issue is the breakdown the bulk-boundary correspondence (BBC) in $\mathrm{NH}$ systems that has recently become a subject of active and controversial discussion 15, 19, 20, 22 25, 31, 50]. Due to the existence of NH skin effect, the conventional approach of predicting boundary states from bulk topological invariants for periodic systems does not provide a conclusive physical picture. According to Ref.[22], it was known that it is non-Bloch topological invariant that characterizes the topological properties of the NH topological systems. However, the non-Bloch topological invariants cannot predict the existence of the (singular) defective edge state (an edge state on the ends of an one-dimensional (1D) topological system with $\mathrm{NH}$ coalescence).

Hence, to complete solve the open issue of the breakdown the bulk-boundary correspondence in NH systems,

${ }^{*}$ Corresponding author; Electronic address: spkou@bnu.edu.cn we develop a new theory for non-Hermitian topological system by proposing the state-dependent topological invariants. We point out that it is the statedependent topological invariants rather than a global state-independent topological invariant that characterize the non-Hermitian topological phases. With the help of effective edge Hamiltonian, we show spontaneous EP phenomenon together with topological Hermitian-NH transition for a given edge state. In addition, due to the unbalance of the state-dependent topological invariants for the edge states on chemical potential there exists spontaneous topological current for 2D non-Hermitian Chern insulator.

State-dependent topological invariants for $1 D \mathrm{NH}$ topological insulator: Firstly, we take 1D nonreciprocal SuSchrieffer-Heeger (SSH) model as an example to introduce the state-dependent topological invariants and provide a new description of bulk-boundary correspondence for 1D NH topological system.

The Bloch Hamiltonian for a nonreciprocal SSH model under periodic boundary condition $(\mathrm{PBC})$ is $H_{\mathrm{PBC}}=$ $\left(t_{1}+t_{2} \cos k\right) \sigma_{x}+\left(t_{2} \sin k+i \gamma\right) \sigma_{y}+\varepsilon \sigma_{z}$ where $t_{1}$ and $t_{2}$ describe the intra-cell and inter-cell hopping strengths, respectively. $\varepsilon$ is the staggered potential and $\gamma$ describes the unequal intra-cell hoppings. $\sigma_{i}$ 's are the Pauli matrices acting on the (A or B) sublattice subspace. In this paper, we set $t_{2}=1$. It was known that due to the $\mathrm{NH}$ skin effect the bulk spectrum of the system becomes that of a NH Hamiltonian $H_{\mathrm{OBC}}$ with open boundary condition (OBC). As a result, the effective bulk Hamiltonian turns into 22, 23] $H_{\mathrm{OBC}}(k)=\left(\bar{t}_{1}+\bar{t}_{2} \cos k\right) \sigma_{x}+\left(\bar{t}_{2} \sin k\right) \sigma_{y}+\varepsilon \sigma_{z}$ where the effective hopping parameters become $\bar{t}_{1}=$ $\sqrt{\left(t_{1}-\gamma\right)\left(t_{1}+\gamma\right)}$, and $\bar{t}_{2}=t_{2}$. Here, $\hat{\mathcal{S}}_{\mathrm{NHP}}$ is a similartransformation, i.e., $|\psi(k)\rangle \rightarrow|\bar{\psi}(k)\rangle=\left|\psi\left(k-i q_{0}\right)\right\rangle=$ $\hat{\mathcal{S}}_{\mathrm{NHP}}|\psi(k)\rangle$ or $|n\rangle \rightarrow|\bar{n}\rangle=e^{-q_{0}(n-1)}|n\rangle$ ( $n$ denotes the cell number) with $e^{q_{0}}=\sqrt{\frac{t_{1}-\gamma}{t_{1}+\gamma}}$.

To completely characterize the edge states, we in- 
troduce the state-dependent topological invariants $\left\{\bar{v}_{L}\right.$, $\left.\bar{v}_{R}\right\}=\left\{\bar{v}_{\xi}, \xi=L, R\right\}$ where $\bar{v}_{L}$ and $\bar{v}_{R}$ are topological invariants for the edge states at left and right, respectively. $\left\{\bar{v}_{L}, \bar{v}_{R}\right\}$ are combination of Bloch topological invariants from $H_{\mathrm{PBC}}$ and non-Bloch topological invariants from $H_{\mathrm{OBC}}$, i.e.,

$$
\bar{v}_{L}=\bar{w} \cdot v_{L}, \bar{v}_{R}=\bar{w} \cdot v_{R}
$$

where $v_{L}=\frac{1}{2 \pi} \int d k \cdot \partial_{k} \varphi_{+}$and $v_{R}=\frac{1}{2 \pi} \int d k \cdot \partial_{k} \varphi_{-}$ are the Bloch winding number that are defined from the Hamiltonian under PBC $H_{\mathrm{PBC}} \cdot \varphi_{ \pm}=\operatorname{Arg}\left(h_{ \pm}\right)$and $h_{ \pm}$is described by $h_{ \pm}=h_{x} \pm i h_{y}\left(h_{x}=t_{1}+t_{2} \cos k\right.$, $\left.h_{y}=t_{2} \sin k+i \gamma\right) ; \bar{w}=\frac{1}{2 \pi} \int_{-\pi}^{\pi} \partial \bar{\phi}(k) d k$ is the non-Bloch topological invariant that is defined from the Hamiltonian under OBC $H_{\mathrm{OBC}}$, where $\bar{\phi}(k)=\tan ^{-1}\left(\bar{h}_{y} / \bar{h}_{x}\right)$ and $\bar{h}_{x}=\bar{t}_{1}+\bar{t}_{2} \cos k, \bar{h}_{y}=\bar{t}_{2} \sin k$.

The state-dependent topological invariants $\left\{\bar{v}_{\xi}, \xi=\right.$ $L, R\}$ become a complete description of $\mathrm{BBC}$ for $1 \mathrm{D}$ NH topological systems: There exist $\left|\bar{v}_{L}\right|$ edge states at left end and $\left|\bar{v}_{R}\right|$ edge states at right end. The statedependent topological invariants are not applied to the case of $\varepsilon=0$. The non-Hermitian SSH model with $\varepsilon=0$ is very special and unstable to arbitrary perturbation breaking chiral symmetry. We will discuss the case of $\varepsilon=0$ in supplementary materials in detail.

As a result, for the 1D NH SSH model, we have a state-dependent phase diagram with four phases (See Fig.1(a)): phase I, phase II, phase III, phase IV. There exist two kinds of topological phase transitions: the stateindependent topological transition at $\left|\bar{t}_{1}\right|=\left|\bar{t}_{2}\right|$ is characterized by the changing of non-Bloch topological invariant $\bar{w}$ for $H_{\mathrm{OBC}}$ from a trivial phase with $\bar{v}_{L}=\bar{v}_{R}=0$ (or $\bar{w}=0$ ) to topological phase with $\bar{v}_{L} \neq$ or $\bar{v}_{R} \neq 0$ (or $\bar{w}=1$ ); the other at $t_{1} \pm \gamma= \pm 1$ is state-dependent that is characterized by the changing of Bloch topological invariant $v_{L}$ or $v_{R}$ for $H_{\mathrm{PBC}}$ from a topological phase with the edge states $\left(\left|\bar{v}_{L} \cdot \bar{v}_{R}\right|=1\right.$, and $\left.\bar{w}=1\right)$ to another without them $\left(\bar{v}_{L} \cdot \bar{v}_{R}=0\right.$, and $\left.\bar{w}=1\right)$.

To verify the validity of the state-dependent topological invariants $\left\{\bar{v}_{\xi}, \xi=L, R\right\}$ and explore the corresponding topological transitions for the 1D NH topological insulators, we write down the effective Hamiltonian for the edge states $\hat{\mathcal{H}}_{\text {edge }}=\left(\begin{array}{ll}h_{11} & h_{12} \\ h_{21} & h_{22}\end{array}\right)$ where $h_{I J}=\left\langle\mathrm{b}^{I}\left|\hat{H}_{\mathrm{NH}}\right| \mathrm{b}^{J}\right\rangle, I, J=1,2 . \quad\left(\mid \begin{array}{l}\left.\mathrm{b}^{1}\right\rangle \\ \left.\mathrm{b}^{2}\right\rangle\end{array}\right)$ are the basis under biorthogonal set of the edge states at left/right ends. For the NH SSH model with $\gamma \neq 0$, the effective edge Hamiltonian is obtained as

$$
\hat{\mathcal{H}}_{\text {edge }}=\bar{\Delta}^{+} \tau^{+}+\bar{\Delta}^{-} \tau^{-}+\varepsilon \tau^{z}
$$

where $\Delta^{+}=\bar{\Delta} e^{-N q_{0}}=\frac{\left(t_{1}^{2}-t_{2}^{2}-\gamma^{2}\right)}{t_{2}}\left(-\frac{t_{1}^{2}-\gamma^{2}}{t_{2}^{2}} e^{-2 q_{0}}\right)^{N / 2}$ and $\Delta^{-}=\bar{\Delta} e^{N q_{0}}=\frac{\left(t_{1}^{2}-t_{2}^{2}-\gamma^{2}\right)}{t_{2}}\left(-\frac{t_{1}^{2}-\gamma^{2}}{t_{2}^{2}} e^{2 q_{0}}\right)^{N / 2} . \bar{\Delta}$ is the energy tunneling with the exponential decay of number of unit cells $N$. In thermodynamic limit $N \rightarrow \infty$, although $\bar{\Delta} \rightarrow 0$, the results are non-trivial.

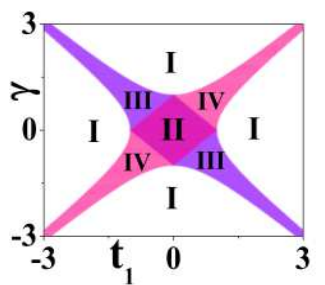

(a)

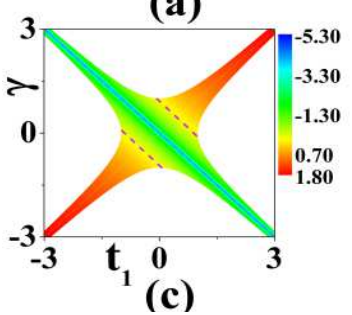

(c)

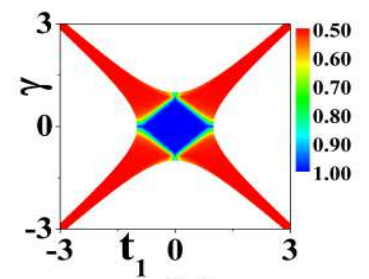

(b)

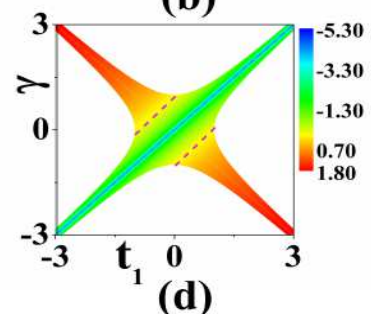

FIG. 1: (Color online) (a) State-dependent phase diagram: phase I with $\bar{v}_{L}=0$ and $\bar{v}_{R}=0$ - trivial phase without edge states; phase II with $\bar{v}_{L}=1$ and $\bar{v}_{R}=1$ - topological phase with two edge states at left and right ends; phase III with $\bar{v}_{L}=1$ and $\bar{v}_{R}=0$ - topological phase with only one edge state at left end; phase IV with $\bar{v}_{L}=0$ and $\bar{v}_{R}=1-$ topological phase with only one edge state at right end; (b) The numerical results for $\mathrm{BBC}$ ratio $\gamma_{\mathrm{BBC}}=1-\frac{\left|\left\langle\psi_{+} \mid \psi_{-}\right\rangle\right|}{2}$, for the case of $N=100, \varepsilon=0.1$. In blue region we have a Hermitian phase. In red regions, we have non-Hermitian phases with spontaneous EP phenomenon. Between the blue region and red regions, topological Hermitian- $\mathrm{NH}$ transition occurs; (c) and (d): The numerical results for off-diagonal term of the effective edge Hamiltonian $\hat{\mathcal{H}}_{\text {edge }}, \Delta^{+}(\mathrm{a})$ and $\Delta^{-}$ (b). The dotted lines denote the topological Hermitian-nonHermitian transition with $\frac{\ln \bar{\Delta}^{ \pm}}{N}=0$ shown in (a) and (b).

According to the off-diagonal term $\Delta^{+}=\bar{\Delta} e^{-N q_{0}}$ or $\Delta^{-}=\bar{\Delta} e^{N q_{0}}$ of $\hat{\mathcal{H}}_{\text {edge }}$, there exists the competition between the exponential decay of $N$ from energy tunneling $\bar{\Delta} \sim e^{\frac{N}{2} \ln \left(-\frac{t_{1}^{2}-\gamma^{2}}{t_{2}^{2}}\right)}$ and the exponential increase with $N$ from NH similarity transformation $e^{ \pm N q_{0}}$. Therefore, in thermodynamic limit (or $N \rightarrow \infty$ ) there exist two phases: one is Hermitian phase with $\left|\bar{\Delta} e^{ \pm N q_{0}}\right| \rightarrow 0$, the other is $\mathrm{NH}$ phase with $\left|\bar{\Delta} e^{ \pm N q_{0}}\right| \rightarrow \infty$. In the Hermitian phase with $\left|\bar{\Delta} e^{ \pm N q_{0}}\right| \rightarrow 0$, the effective edge Hamiltonian is reduced into $\hat{\mathcal{H}}_{\text {edge }} \rightarrow \varepsilon \cdot \tau^{z}$. Now, the effect from the $\mathrm{NH}$ similarity transformation is irrelevant; On the other hand, in the NH phase with $\left|\bar{\Delta} e^{ \pm N q_{0}}\right| \rightarrow \infty$, the effective edge Hamiltonian is reduced into $\hat{\mathcal{H}}_{\text {edge }} \rightarrow \bar{\Delta} e^{-N q_{0}} \tau^{+}$or $\bar{\Delta} e^{N q_{0}} \tau^{-}$. Now, the effect from NH similarity transformation dominates and becomes relevant. Although the total energy splitting $E_{+}-E_{-}=2 \sqrt{\varepsilon^{2}+\bar{\Delta}^{2}}$ is finite, the system is at exceptional points (EPs) and we have singular defective edge state with $\mathrm{NH}$ coalescence. For this reason, we call it spontaneous EP phenomenon.

At $\left|\bar{\Delta} e^{ \pm q_{0}}\right|=1$, the topological transition between Hermitian phase and NH phase with spontaneous EP occurs. We call the state-dependent topological transition to be topological Hermitian-NH transition. Fig.1(c) and 
Fig.1(d) are the numerical results for $\Delta^{+}=\bar{\Delta} e^{-N q_{0}}$ or $\Delta^{-}=\bar{\Delta} e^{N q_{0}}$, in which $\frac{\ln \Delta^{ \pm}}{N}=0$ or $\left|\frac{t_{1}^{2}-\gamma^{2}}{t_{2}^{2}} e^{ \pm 2 q_{0}}\right|=1$ denotes the topological Hermitian-NH transition. This condition for topological Hermitian-NH transition is just $t_{1} \pm \gamma= \pm 1$ from phase with the edge states $\left(\left|\bar{v}_{L} \cdot \bar{v}_{R}\right|=1\right.$, and $\bar{w}=1)$ to another without them $\left(\bar{v}_{L} \cdot \bar{v}_{R}=0\right.$, and $\bar{w}=1$ ). To verify this type of topological transitions and show the defectiveness of the edge states, we calculate the $\mathrm{BBC}$ ratio, $\gamma_{\mathrm{BBC}}=1-\frac{\left|\left\langle\psi_{+} \mid \psi_{-}\right\rangle\right|}{2}$, a quantity that characterizes number anomaly of the edge states. If $\Upsilon_{\mathrm{BBC}}$ is 1 , there exists the usual BBC with $\bar{v}_{L}=1$ and $\bar{v}_{R}=1$; if $\Upsilon_{\mathrm{BBC}}$ is smaller than 1 , there exists defective edge states with $\bar{v}_{L}=1$ and $\bar{v}_{R}=0$ or $\bar{v}_{L}=0$ and $\bar{v}_{R}=1$. In Fig.1(b), we show the numerical and analytic results that are consistent with each other.

State-dependent topological invariants and spontaneous topological current for $2 \mathrm{D} \mathrm{NH}$ topological insulators: Next, we consider a lattice model of 2D NH Chern insulator - the 2D NH spin-orbital coupling model[4]. The Bloch Hamiltonian under $\mathrm{PBC}$ is $H_{\mathrm{PBC}}\left(k_{x}, k_{y}\right)=$ $\left(\sin k_{x}\right) \sigma_{x}+\left(\sin k_{y}+i \gamma\right) \sigma_{y}+\left(m+\cos k_{x}+\cos k_{y}\right) \sigma_{z}$, where $\sigma_{x, y, z}$ are Pauli matrices. The NH parameters $\gamma$ appear as "imaginary Zeeman field". Suppose that the cylinder has periodic-boundary condition along $x$-direction and open boundary condition along $y$-direction, by doing similar transformation $\hat{\mathcal{S}}_{\mathrm{NHP}},|\psi(k)\rangle \rightarrow|\bar{\psi}(k)\rangle=$ $\left|\psi\left(k-i q_{0}\right)\right\rangle=\hat{\mathcal{S}}_{\mathrm{NHP}}|\psi(k)\rangle$, we have the non-Bloch "cylinder Hamiltonian", $H_{\mathrm{OBC}, y}\left(k_{x}, \tilde{k}_{y}\right)=H_{\mathrm{PBC}}\left(k_{x} \rightarrow\right.$ $k_{x}, k_{y} \rightarrow \tilde{k}_{y}-i q_{0}$, in which $e^{-2 q_{0}\left(k_{x}\right)}=\frac{m+\cos k_{x}+\gamma}{m+\cos k_{x}-\gamma}$.

Then, we define the state-dependent topological invariants for edge states in the 2D NH spin-orbital coupling model. For OBC along $y$-direction and $\mathrm{PBC}$ along $x$ direction, the topological system exhibits modes localized on the edges and the wave vectors $k_{x}=k=\frac{2 \pi n}{L_{x}}$ $\left(n=1,2, \ldots, N_{x}\right)$ are good quantum numbers. The statedependent topological invariants are defined as

$$
\left\{\bar{v}_{\xi, k}, k \in T^{1}, k \neq 0, \xi=L, R\right\}
$$

where $\bar{v}_{k, L}=\mathcal{C}_{y} \cdot v_{k, L}$ are topological invariants for all edge state at left end with wave vector $k$ and $\bar{v}_{k, R}=\mathcal{C}_{y} \cdot v_{k, R}$ are topological invariants for all edge state at right end with wave vector $k$, respectively. Here, $\mathcal{C}_{y}=\frac{1}{2 \pi i} \int d k_{x} d \tilde{k}_{y} \epsilon^{i j}\left\langle\partial_{i} u_{L}\left(k_{x}, \tilde{k}_{y}\right) \mid \partial_{j} u_{R}\left(k_{x}, \tilde{k}_{y}\right)\right\rangle$ is the non-Bloch Chern number that is defined from the Hamiltonian $H_{\mathrm{OBC}, y}\left(k_{x}, \tilde{k}_{y}\right) \quad\left(\left|u_{R}\left(k_{x}, \tilde{k}_{y}\right)\right\rangle\right.$ denotes the bulk state of biorthogonal set under OBC); $v_{L, k}=$ $\frac{1}{2 \pi} \int d k_{y} \partial_{k_{y}} \varphi_{+}\left(k_{y}, k\right)$ and $v_{R, k}=\frac{1}{2 \pi} \int d k_{y} \partial_{k_{y}} \varphi_{-}\left(k_{y}, k\right)$ are the Bloch winding number that are defined from the Hamiltonian $H_{\mathrm{PBC}}\left(k_{x}, k_{y}\right) . \quad h_{ \pm}$is described by $h_{ \pm}=$ $h_{x} \pm i h_{y}\left(h_{x}=(m+\cos k)+\cos k_{y}, h_{y}=\sin k_{y}+i \gamma\right)$ and $\varphi_{ \pm}=\operatorname{Arg}\left(h_{ \pm}\right)$.

Therefore, the state-dependent topological invariants $\left\{\bar{v}_{\xi, k}, k \in T^{1}, k \neq 0, \xi=L, R\right\}$ becomes a complete description of $\mathrm{BBC}$ for $2 \mathrm{D} \mathrm{NH}$ topological systems: There exist $\left|\bar{v}_{L, k}\right|$ edge states with wave vector $k$ at left end and $\left|\bar{v}_{R, k}\right|$ edge states with wave vector $k$ at righ end. The state-dependent topological invariants are not applied to

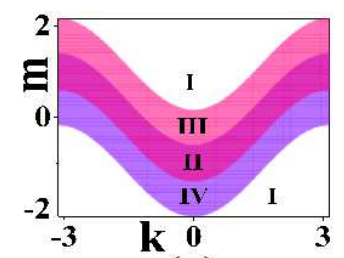

(a)

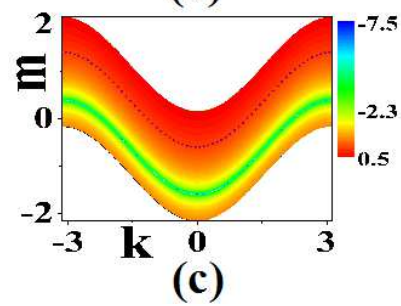

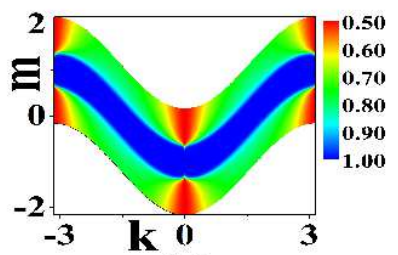

(b)

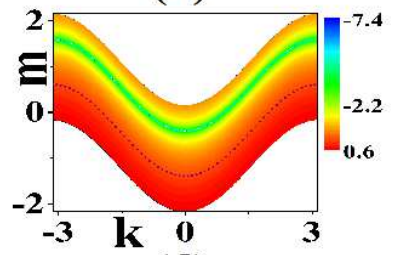

(d)
FIG. 2: (Color online) (a) is the state-dependent phase diagram via $m$ and $k$ with fixed $\gamma=0.6$ : phase I with $\bar{v}_{L, k}=0$ and $\bar{v}_{R, k}=0$ - trivial phase without edge states; phase II with $\bar{v}_{L, k}=1$ and $\bar{v}_{R, k}=1$ - topological phase with two edge states at left and right ends; phase III with $\bar{v}_{L, k}=1$ and $\bar{v}_{R, k}=0-$ topological phase with only one edge state at left end; phase IV with $\bar{v}_{L, k}=0$ and $\bar{v}_{R, k}=1$ - topological phase with only one edge state at right end; (b) The numerical results for BBC ratio with fixed $\gamma=0.6, \gamma_{k, \mathrm{BBC}}=1-\frac{\left|\left\langle\psi_{k,+} \mid \psi_{k,-}\right\rangle\right|}{2} ;$ (c) and (d): The numerical results for off-diagonal term of the effective edge Hamiltonian $\hat{\mathcal{H}}_{\text {edge }}$ for the case of $\gamma=0.6, \Delta_{k}^{+}$(c) and $\Delta_{k}^{-}(\mathrm{d})$. The dotted lines correspond to $\frac{\ln \bar{\Delta}_{k}^{ \pm}}{N}=0$, the topological Hermitian-non-Hermitian transition shown in (a) and (b).

the case of $k=0$ and we will discuss the case of $k=0$ in supplementary materials in detail. As a result, for the 2D NH topological system with fixed parameter, we have a state-dependent phase diagram via $k$ and $m$ (See Fig.2(a)).

To verify the validity of the state-dependent topological invariants, we obtain the effective edge Hamiltonian for the NH spin-orbital coupling model.

Firstly, the effective edge Hamiltonian of edge states for Hermitian 2D Chern insulator with $\gamma=0$ is obtained as $\hat{\mathcal{H}}_{\text {edge }}=\tau^{z} \varepsilon_{k}+\tau^{x} \Delta_{k}$, where $\varepsilon_{k}=\sin k$ is the dispersion of the edge states of semi-infinite system and $\Delta_{k}=\left((\cos k+m)^{2}-1\right) \cdot(\cos k+m)^{N_{y}}$ is tunneling strength. As a result, the energy levels are $E_{k}= \pm \sqrt{(\sin k)^{2}+\left(\Delta_{k}\right)^{2}}$. In thermodynamic limit $N_{y} \rightarrow \infty, \Delta_{k} \rightarrow 0$, we have $E_{k} \rightarrow \pm \sin k$ (or $E_{k} \rightarrow \sin k$ on left/right edge and $E_{k} \rightarrow-\sin k$ on right/left edge).

When considering the $\mathrm{NH}$ skin effect, we do an additional $\mathrm{NH}$ similarity transformation $U_{\text {edge }}=$ $\left(\begin{array}{cc}1 & 0 \\ 0 & e^{-q_{0}(k) N_{y}}\end{array}\right)$ on the effective edge Hamiltonian $\hat{\mathcal{H}}_{\text {edge }}$, i.e., $\tau^{x} \rightarrow U_{\text {edge }}^{-1} \tau^{x} U_{\text {edge }}=\tau^{x} \cosh \left(q_{0}(k) N_{y}\right)-$ $i \tau^{y} \sinh \left(q_{0}(k) N_{y}\right)$. As a result, the effective edge Hamiltonian turns into

$$
\hat{\mathcal{H}}_{\text {edge }}=\bar{\Delta}_{k}^{+} \tau^{+}+\bar{\Delta}_{k}^{-} \tau^{-}+\tau^{z} \sin k
$$


where $\bar{\Delta}_{k}^{+}=\bar{\Delta}_{k} e^{-N_{y} q_{0}(k)}, \bar{\Delta}_{k}^{-}=\bar{\Delta}_{k} e^{N_{y} q_{0}(k)}$ and $\bar{\Delta}_{k}=$ $\left((\cos k+m)^{2}-1-\gamma^{2}\right) \cdot\left(\gamma^{2}-(\cos k+m)^{2}\right)^{N_{y} / 2}$. Under $U_{\text {edge }}$, the energy levels become $E_{ \pm}(k)= \pm \sqrt{(\sin k)^{2}+\left(\bar{\Delta}_{k}\right)^{2}}$.

With the help of the effective edge Hamiltonian $\hat{\mathcal{H}}_{\text {edge }}$, we show spontaneous EP phenomenon and topological Hermitian-NH transition for a given edge state with wave vector $k$. At $\frac{\ln \bar{\Delta}_{k}^{ \pm}}{N}=0$ topological Hermitian$\mathrm{NH}$ transition occurs that is just the condition of $\left|\left((\cos k+m)^{2}-\gamma^{2}\right) e^{ \pm 2 q_{0}}\right|=1$ from phase with the edge states $\left(\left|\bar{v}_{L, k} \cdot \bar{v}_{R, k}\right|=1\right.$, and $\left.\mathcal{C}_{y}=1\right)$ to another without them $\left(\bar{v}_{L, k} \cdot \bar{v}_{R, k}=0\right.$, and $\left.\mathcal{C}_{y}=1\right)$. Fig.2(c) and Fig.2(d) are the numerical results for $\bar{\Delta}_{k}^{+}$or $\bar{\Delta}_{k}^{-}$, in which $\frac{\ln \bar{\Delta}_{k}^{ \pm}}{N}=0$ denotes the critical points of the topological transitions. We can also use $\Upsilon_{\mathrm{BBC}}$ to characterize the topological properties of the edge states with wave vector $k, \Upsilon_{k, \mathrm{BBC}}=1-\left|\left\langle\psi_{k,+} \mid \psi_{k,-}\right\rangle\right| / 2$. See the results in Fig.2(b).

In addition, we point out that there exists physics consequence of unbalance of the state-dependent topological invariants for the edge states on chemical potential - the spontaneous topological current for 2D NH Chern insulator.

For the system with open boundary condition (OPC), in general, the chemical potentials at the ends of system may be different, i.e., $\mu_{L}$ and $\mu_{R}$, respectively. We assume that the chemical potentials locate inside the energy gap of the bulk states. So, the transport of the system mainly comes from the edge states and we can apply the Landauer-Buttiker formalism on the transport of edge states. According to the Landauer-Buttiker formalism, the (Hall) current is defined as $I_{H}=-e v_{\mathrm{eff}} \cdot n$. In thermodynamic limit, the effective velocity of the charge carriers is $v_{\text {eff }}=\frac{1}{\hbar} \frac{\partial E_{k}}{\partial k}$ where $E_{k} \sim \pm \sin k$. The density of the charge carriers is

$$
\begin{aligned}
n & =n_{L}-n_{R}=\mu_{L} \mathcal{D}\left(\mu_{L}\right)-\mu_{R} \mathcal{D}\left(\mu_{R}\right) \\
& \sim\left(\mu_{L} \nu_{L, k_{L}}(E)-\mu_{R} \nu_{R, k_{R}}(E)\right) D(E)
\end{aligned}
$$

where $\mathcal{D}\left(\mu_{L}\right)=\nu_{L, k_{L}}(E) D(E)$ and $\mathcal{D}\left(\mu_{R}\right)=$ $\nu_{R, k_{R}}(E) D(E) . \nu_{L, k_{L}}(E)$ and $v_{R, k_{R}}(E)$ are the statedependent topological invariants for the edge states at left and right sides, respectively. The wave vectors $k_{L}$ and $k_{R}$ are obtained by calculating the following equations, $\sin k_{L}=\mu_{L}$ and $-\sin k_{R}=\mu_{R}$, respectively. As a result, considering $\mu_{L / R}=-e V_{L / R}$, we derive the current for the NH 2D topological insulator,

$$
\begin{aligned}
I_{H} & =\frac{e^{2}}{h} \mathcal{C}_{y}\left(V_{L} \nu_{L, k_{L}}(E)-V_{R} \nu_{R, k_{R}}(E)\right) \\
& =\sigma_{0}\left(V_{L} \bar{\nu}_{L, k_{L}}(E)-V_{R} \bar{\nu}_{R, k_{R}}(E)\right)
\end{aligned}
$$

where $\sigma_{0}=\frac{e^{2}}{h}$ is unit of quantized Hall conductance.

In phase III and phase IV of Fig.2(a), when there doesn't exist an external transverse electric field $\mu_{L}=$ $\mu_{R}=\mu$, due to the unbalance of the state-dependent topological invariants for the edge states on chemical potential, the electric current still exists, i.e., $I_{H}=$ $\sigma_{0} \mu\left(\bar{v}_{L, k_{L}}-\bar{v}_{R, k_{R}}\right) \neq 0$. Because the current is proportional to the unbalance of the state-dependent topological invariant $\left(\bar{v}_{L, k_{L}}-\bar{v}_{R, k_{R}}\right)$, the spontaneous current is topological!

State-dependent Topological Invariants for $\mathrm{NH} d$ dimensional topological insulators: Finally, we generalize the theory of state-dependent topological invariants to $\mathrm{NH} d$-dimensional topological insulators.

A $d$-dimensional topological system can be approximatively described by continuum model. Assuming PBCs in all directions, we consider the following Hamiltonian for $\mathrm{NH} d$-dimensional topological insulators as $H_{\mathrm{PBC}}(\mathbf{k})=\sum_{i=1}^{d} k_{i} \Gamma_{i}+\left(m-\frac{1}{2} \sum_{i=1}^{d} k_{i}^{2}\right) \Gamma_{d+1}+i \gamma \Gamma_{j}$, where $\Gamma_{\mu}$ denote the gamma matrices that satisfy $\left\{\Gamma_{\mu}, \Gamma_{\nu}\right\}=2 \delta_{\mu \nu}$ and $m$ is the real mass parameter. $\gamma$ denotes the strength of the $\mathrm{NH}$ term. With OBCs in the $j$-th direction and PBCs in all other directions, due to the residue translation symmetry, $\tilde{\mathbf{k}}$ denotes the vector of all momenta except $k_{j}$. Under a similar transformation $\hat{\mathcal{S}}_{\mathrm{NHP}}=\operatorname{diag}\left\{1, \alpha, \cdots, \alpha^{N_{j}-1}\right\} \otimes$ $\left[(1+\alpha) \mathbb{I}+i(1-\alpha) \Gamma_{j} \Gamma_{d+1}\right]$, the Hamiltonian is transformed to $H_{\mathrm{OBC}}(\tilde{\mathbf{k}})=\sum_{i \neq j} k_{i} \Gamma_{i}+\sqrt{M_{k}^{2}-\gamma^{2}} \Gamma_{d+1}$, where $\alpha=\sqrt{\left(M_{k}-\gamma\right) /\left(M_{k}+\gamma\right)}, M_{k}=m-\frac{1}{2} \sum_{i \neq j} k_{i}^{2}$, and $N_{j}$ stands for the number of layers in the $j$-th direction.

Then, to completely characterize the topological properties, we define the state-dependent topological invariants for edge states in the $d$-D NH topological model.

With OBCs in the $j$-th direction and PBCs in all other directions, due to the residue translation symmetry, there exist edge states on left/right edges with wave vector $\tilde{\mathbf{k}}$. The state-dependent topological invariants for edge states are defined as

$$
\left\{\bar{v}_{\xi, \tilde{\mathbf{k}}}, \tilde{\mathbf{k}} \in T^{d-1}, \tilde{\mathbf{k}} \neq 0, \text { and } \xi=L, R\right\}
$$

where $\bar{v}_{L, \tilde{\mathbf{k}}}=\mathcal{W}_{j} \cdot v_{L, \tilde{\mathbf{k}}}$ for edge state at left end with wave vector $\tilde{\mathbf{k}}$ and $\bar{v}_{R, \tilde{\mathbf{k}}}=\mathcal{W}_{j} \cdot v_{R, \tilde{\mathbf{k}}}$ for the edge state at right end with wave vector $\tilde{\mathbf{k}}$. Here $\mathcal{W}_{j}$ is the d-dimensional non-Bloch topological invariant that is defined from the Hamiltonian $H_{\mathrm{OBC}}(\tilde{\mathbf{k}}) . \quad v_{L, \tilde{\mathbf{k}}}$ and $v_{R, \tilde{\mathbf{k}}}$ are the onedimensional winding number that are defined from the Hamiltonian $H_{\mathrm{PBC}}(\mathbf{k}) . \quad v_{L, \tilde{\mathbf{k}}}=\frac{1}{2 \pi} \int d k_{j} \partial_{k_{j}} \varphi_{+}\left(k_{j}, \tilde{\mathbf{k}}\right)$ and $v_{R, \tilde{\mathbf{k}}}=\frac{1}{2 \pi} \int d k_{j} \partial_{k_{j}} \varphi_{-}\left(k_{j}, \tilde{\mathbf{k}}\right)$ are the Bloch winding number that are defined from the Hamiltonian under closed boundary condition. $h_{ \pm}$is described by $h_{ \pm}=h_{x} \pm i h_{y}\left(h_{x}=\sum_{i=1}^{d} \frac{1}{2} k_{i}^{2}, h_{y}=k_{j}+i \gamma\right)$ and $\varphi_{ \pm}=\operatorname{Arg}\left(h_{ \pm}\right)$. The topological Hermitian-NH transition occurs at $m-\sum_{i \neq j}^{d} \frac{1}{2} k_{i}^{2}= \pm \gamma \pm 1$.

Therefore, the state-dependent topological invariants $\left\{\bar{v}_{\xi, \tilde{\mathbf{k}}}, \tilde{\mathbf{k}} \in T^{d-1}, \tilde{\mathbf{k}} \neq 0\right.$, and $\left.\xi=L, R\right\}$ become a complete description of BBC for $d$-D NH topological systems: There exist $\left|\bar{v}_{R, \tilde{\mathbf{k}}}\right|$ edge states with wave vector $\tilde{\mathbf{k}}$ at left end and $\left|\bar{v}_{L, \tilde{\mathbf{k}}}\right|$ edge states with wave vector $\tilde{\mathbf{k}}$ at right end. The state-dependent topological invariants are not 
applied to the case of $\tilde{\mathbf{k}}=0$ and we will discuss the case of $\tilde{\mathbf{k}}=0$ in supplementary materials in detail.

Conclusion and discussion: In the end, we draw a brief conclusion. The theory of state-dependent topological invariants for $\mathrm{NH}$ topological insulators is developed. The key point of our theory is that each edge state is characterized by state-dependent topological invariants $\left\{\bar{v}_{\xi, \tilde{\mathbf{k}}}\right.$, $\tilde{\mathbf{k}} \in T^{d-1}, \tilde{\mathbf{k}} \neq 0$, and $\left.\xi=L, R\right\}$ rather than a global (state-independent) non-Bloch topological invariant $w_{j}$. To completely characterize the edge states in a NH topological systems, one need to calculate all state-dependent topological invariants. With the help of effective edge Hamiltonian $\hat{\mathcal{H}}_{\text {edge }}$, we derive the state-dependent phase diagram and show spontaneous EP phenomenon together with (state-dependent) topological Hermitian- $\mathrm{NH}$ transition for a given edge state with given wave vector $\tilde{\mathbf{k}}$. In addition, there exists spontaneous topological current $I_{H}$ for 2D NH Chern insulator that is proportional to the unbalance of the state-dependent topological invariants $\left(\bar{v}_{L, k_{L}}-\bar{v}_{R, k_{R}}\right)$ for the edge states on chemical potential $\mu_{L}=\mu_{R}=\mu$. In future, the theory of state-dependent topological invariants can be applied to other types of topological systems, including topological superconductors, higher order topological states, even the topological semi-metals.

\section{Acknowledgments}

This work is supported by NSFC Grant No. 11674026 , 11974053. We thank Ya-Jie Wu, and Gao-Yong Sun for their helpful discussion.
[1] M. Z. Hasan and C. L. Kane, Rev. Mod. Phys. 82, 3045 (2010).

[2] X.-L. Qi and S.-C. Zhang, Rev. Mod. Phys. 83, 1057 (2011).

[3] D. J. Thouless, M. Kohmoto, M. P. Nightingale, and M. den Nijs, Phys. Rev. Lett. 49, 405.

[4] X. L. Qi, Y. S. Wu, and S. C. Zhang, Phys. Rev. B 74, 085308 (2006).

[5] F. D. M. Haldane, Phys. Rev. Lett. 61, 2015 (1988).

[6] C. L. Kane and E. J. Mele, Phys. Rev. Lett. 95, 226801 (2005); Phys. Rev. Lett. 95, 146802 (2005).

[7] J. Alicea, Rep. Prog. Phys. 75, 076501 (2012).

[8] C.-K. Chiu, J. C. Y. Teo, A. P. Schnyder, and S. Ryu, Rev. Mod. Phys. 88, 035005 (2016).

[9] A. Bansil, H. Lin, and T. Das, Rev. Mod. Phys. 88, 021004(2016).

[10] M. S. Rudner and L. S. Levitov, Phys. Rev. Lett. 102, 065703 (2009).

[11] K. Esaki, M. Sato, K. Hasebe, and M. Kohmoto, Phys. Rev. B 84, 205128 (2011).

[12] Y. C. Hu and T. L. Hughes, Phys. Rev. B 84, 153101 (2011).

[13] S.-D. Liang and G.-Y. Huang, Phys. Rev. A 87, 012118 (2013).

[14] B. Zhu, R. Lü, and S. Chen, Phys. Rev. A 89, 062102 (2014).

[15] T. E. Lee, Phys. Rev. Lett. 116, 133903 (2016).

[16] Y. Xu, S.-T. Wang, and L.-M. Duan, Phys. Rev. Lett. 118, 045701 (2017)

[17] D. Leykam, K. Y. Bliokh, C. Huang, Y. D. Chong, and F. Nori, Phys. Rev. Lett. 118, 040401 (2017).

[18] H. Shen, B. Zhen, and L. Fu, Phys. Rev. Lett. 120, 146402 (2018).

[19] Y. Xiong, J. Phys. Commun. 2, 035043 (2018).

[20] K. Kawabata, Y. Ashida, H. Katsura, and M. Ueda, Phys. Rev. B 98, 085116 (2018).

[21] Z. Gong, Y. Ashida, K. Kawabata, K. Takasan, S. Higashikawa, and M. Ueda, Phys. Rev. X 8, 031079 (2018).

[22] S. Yao, and Z. Wang, Phys. Rev. Lett. 121, 086803 (2018).

[23] S. Yao, F. Song, and Z. Wang, Phys. Rev. Lett.

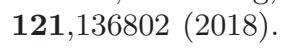

[24] F. K. Kunst, E. Edvardsson, J. C. Budich, and E. J. Bergholtz, Phys. Rev. Lett. 121, 026808 (2018).

[25] C. Yin, H. Jiang, L. Li, R. Lü, and S. Chen, Phys. Rev. A 97, 052115 (2018).

[26] K. Kawabata, K. Shiozaki, and M. Ueda, Phys. Rev. B 98, 165148 (2018).

[27] V. M. M. Alvarez, J. E. B. Vargas, M. Berdakin, and L. E. F. F. Torres, Eur. Phys. J. Spec. Top. 227, 1295 (2018).

[28] H. Jiang, C. Yang, and S. Chen, Phys. Rev. A 98, 052116 (2018).

[29] A. McDonald, T. Pereg-Barnea, and A. A. Clerk, Phys. Rev. X 8, 041031 (2018).

[30] H.-G. Zirnstein, G. Refael, and B. Rosenow, arXiv:1901.11241

[31] L. Jin and Z. Song, Phys. Rev. B 99, 081103 (2019); S. Lin, L. Jin, and Z. Song, Phys. Rev. B 99, 165148 (2019); K. L. Zhang, H. C. Wu, L. Jin, and Z. Song, Phys. Rev. B 100, 045141 (2019).

[32] C. H. Lee and R. Thomale, Phys. Rev. B 99, 201103(R) (2019).

[33] T. Liu, Y.-R. Zhang, Q. Ai, Z. Gong, K. Kawabata, M. Ueda, and F. Nori, Phys. Rev. Lett. 122, 076801 (2019).

[34] K. Kawabata, K. Shiozaki, M. Ueda, and M. Sato, Phys. Rev. X 9, 041015 (2019).

[35] H. Zhou and J. Y. Lee, Phys. Rev. B 99, 235112 (2019).

[36] C. H. Liu, H. Jiang, S. Chen, Phys. Rev. B 99, 125103 (2019).

[37] L. Herviou, J. H. Bardarson, and N. Regnault, Phys. Rev. A 99, 052118 (2019).

[38] K. Yokomizo and S. Murakami, Phys. Rev. Lett. 123, 066404 (2019).

[39] R. Chen, C.-Z. Chen, B. Zhou, and D.-H. Xu, Phys. Rev. B 99, 155431 (2019).

[40] T. S. Deng and W. Yi, Phys. Rev. B 100, 035102 (2019).

[41] F. Song, S. Yao, and Z. Wang, Phys. Rev. L 123, 170401 (2019).

[42] F. Song, S. Yao, and Z. Wang, Phys. Rev. Lett. 123, 246801 (2019).

[43] Xi-Wang Luo and Chuanwei Zhang, Phys. Rev. Lett. 123,073601 (2019).

[44] N. Okuma and M. Sato, Phys. Rev. Lett. 123, 097701 
(2019).

[45] E. J. Bergholtz and J. C. Budich, Phys. Rev. Research 1, 012003(R) (2019).

[46] J. Y. Lee, J. Ahn, H. Zhou, and A. Vishwanath, Phys. Rev. Lett. 123, 206404 (2019).

[47] W. B. Rui, M. M. Hirschmann, and A. P. Schnyder, Phys. Rev. B 100, 245116 (2019).

[48] H. Schomerus, Phys. Rev. Research 2, 013058 (2020).

[49] K.-I. Imura and Y. Takane, Phys. Rev. B 100, 165430 (2019).

[50] L. Herviou, N. Regnault, and J. H. Bardarson, SciPost .Phys. 7, 069 (2019).

[51] P.-Y. Chang, J.-S. You, X. Wen, and S. Ryu, arXiv:1909.01346.

[52] X.-X. Zhang and M. Franz, Phys. Rev. Lett. 124, 046401 (2020).

[53] K. Zhang, Z. Yang, and C. Fang, arXiv:1910.01131; Z. Yang, K. Zhang, C. Fang, and J. Hu, arXiv:1912.05499

[54] N. Okuma, K. Kawabata, K. Shiozaki, and M. Sato, Phys. Rev. Lett. 124, 086801 (2020).

[55] S. Longhi, Phys. Rev. Lett. 124, 066602 (2020).

[56] X.-R. Wang, C.-X. Guo, and S.-P. Kou, Phys. Rev. B 101, 121115(R) (2020).

[57] T. Yoshida, T. Mizoguchi, and Y. Hatsugai, arXiv:1912.12022.

[58] K. Kawabata, N. Okuma, and M.Sato, arXiv:2003.07597v1.

[59] C. Wang, X.-R. Wang, C.-X. Guo, and S.-P. Kou, arXiv:2003.10089 1 .

[60] J. M. Zeuner, M. C. Rechtsman, Y. Plotnik, Y. Lumer, S. Nolte, M. S. Rudner, M. Segev, and A. Szameit, Phys. Rev. Lett. 115, 040402 (2015).
[61] S. Weimann, M. Kremer, Y. Plotnik, Y. Lumer, S. Nolte, K. G. Makris, M. Segev, M. C. Rechtsman, and A. Szameit, Nat. Mater. 16, 433 (2017).

[62] L. Xiao, X. Zhan, Z. H. Bian, K. K. Wang, X. Zhang, X. P. Wang, J. Li, K. Mochizuki, D. Kim, N. Kawakami, W. Yi, H. Obuse, B. C. Sanders, and P. Xue, Nat. Phys. 13, 1117 (2017).

[63] H. Zhou, C. Peng, Y. Yoon, C. W. Hsu, K. A. Nelson, L. Fu, J. D. Joannopoulos, M. Soljaci c, and B. Zhen, Science 359, 1009 (2018).

[64] M. A. Bandres, S. Wittek, G. Harari, M. Parto, J. Ren, M. Segev, D. N. Christodoulides, and M. Khajavikhan, ,Science 359, 4005 (2018). G. Harari, M. A. Bandres, Y. Lumer, M. C. Rechtsman, Y. D. Chong, M. Khajavikhan, D. N. Christodoulides, and M. Segev, Science 358, eaar4003 (2018);

[65] A. Cerjan, S. Huang, M. Wang, K. P. Chen, Y. Chong, and M. C. Rechtsman, Nat. Photon. 13, 623 (2019).

[66] K. Wang, X. Qiu, L. Xiao, X. Zhan, Z. Bian, B. C. Sanders, W. Yi, and P. Xue, Nat. Commun. 10, 2293 (2019)

[67] H. Zhao, X. Qiao, T. Wu, B. Midya, S. Longhi, and L. Feng, Science 365, 1163 (2019).

[68] M. Brandenbourger, X. Locsin, and C. Coulais E. Lerner, Nat. Commun. 10, 4608 (2019); A. Ghatak, M. Brandenbourger, J. van Wezel, and C. Coulais, arXiv:1907.11619.

[69] L. Xiao, T. Deng, K. Wang, G. Zhu, Z. Wang, W. Yi, P. Xue, arXiv:1907.12566.

[70] T. Helbig, T. Hofmann, S. Imhof, M. Abdelghany, T. Kiessling, L. W. Molenkamp, C. H. Lee, A. Szameit, M. Greiter, and R. Thomale, arXiv:1907.11562. 